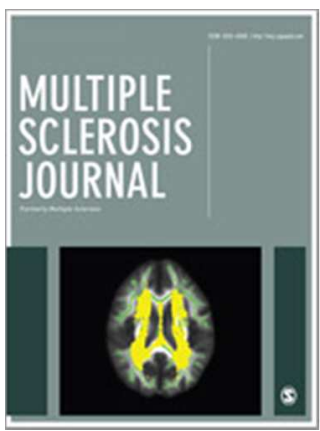

\title{
Cladribine versus fingolimod, natalizumab and interferon $\beta$ for multiple sclerosis
}

\begin{tabular}{|r|l|}
\hline Journal: & Multiple Sclerosis Journal \\
\hline Manuscript ID & MSJ-17-0269.R1 \\
\hline Manuscript Type: & Original Research Paper \\
\hline Date Submitted by the Author: & n/a \\
\hline Complete List of Authors: & Kalincik, Tomas; University of Melbourne, Department of Medicine; Royal \\
& Melbourne Hospital, Department of Neurology \\
& Jokubaitis, Vilija; University of Melbourne, Department of Neurology \\
& Spelman, Tim; University of Melbourne, Department of Neurology \\
& Horakova, Dana; Charles University in Prague, Neurology \\
& Havrdova, Eva; Charles University in Prague, Neurology \\
& Troiano, Maria; Department of Basic Medical Sciences, Neuroscience, and \\
& Sense Organs, University of Bari, Bari, Department of Basic Medical \\
& Sciences, Neuroscience, and Sense Organs \\
& Lechner-Scott, Jeannette; John Hunter Hospital, Neurology \\
& Lugaresi, Alessandra; Universita degli Studi di Bologna Scuola di Medicina e \\
& Chirurgia, Department of Biomedical and Neuromotor Sciences(DIBINEM)- \\
& "Alma Mater Studiorum" \\
& Prat, Alexandre; Université de Montréal, Neuroscience \\
& Girard, Marc; Charles LeMoyne Hospital, Neurology \\
& Duquette, Pierre; Charles LeMoyne Hospital, Neurology \\
& Grammond, Pierre; Hotel Dieu De Levis, Neurology \\
& Solaro, Claudio; PA Micone Hospital, Department of Head and Neck, \\
& Neurology Unit, ASL3 Genovese \\
& Grand'Maison, Francois; Charles LeMoyne Hospital, Neurology \\
& Hupperts, Raymond; Zuyderland Ziekenhuis, Neurology \\
& Prevost, Julie; CSSS Saint-Jérôme, CSSS Saint-Jérôme \\
Sola, Patrizia; University of Modena, Department of Neuroscience, & Neurology section \\
& Ferraro, Diana; Nuovo Ospedale Civile S. Agostino Estense, Neurosciences \\
& Terzi, Murat; Ondokuz Mayis University, Medical School, Neurology \\
& Butler, Ernest; Monash Health, Department of Neurosciences \\
Slee, Mark; Flinders Medical Centre, Department of Neurology \\
Kermode, Allan; Australian Neuromuscular Research Institute, Sir Charles \\
Gairdner Hospital; Centre for, Australian Neuromuscular Research \\
Institute, Sir Charles Gairdner Hospital; Centre for
\end{tabular}


Keywords:

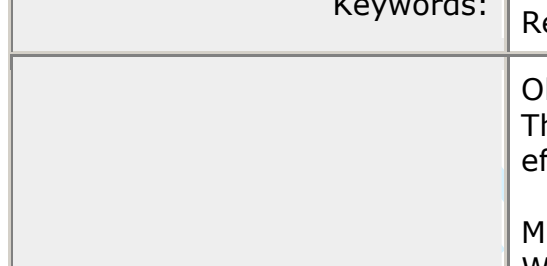

Fabis-Pedrini, Marzena; University of Western Australia, Centre for Neuromuscular and Neurological Disorders

McCombe, Pamela; The University of Queensland, School of Medicine Barnett, Michael; Sydney Neuroimaging Analysis Centre, Sydney Neuroimaging Analysis Centre; University of Sydney, Brain and Mind Research Institute

Shaw, Cameron; Geelong Hospital, Department of Neurology Hodgkinson, Sue; University of New South Wales, Neurology Butzkueven, Helmut; University of Melbourne, Medicine

Disease modifying therapies, Beta-interferon, Fingolimod, Natalizumab, Relapsing/remitting, Second line treatment

Objective

This propensity score-matched analysis from MSBase compared the effectiveness of cladribine with interferon $\beta$, fingolimod or natalizumab.

Methods

We identified all patients with relapse-onset multiple sclerosis, exposure to the study therapies, and $\geq 1$-year on-treatment follow-up from MSBase. Three pairwise propensity score-matched analyses compared treatment outcomes over one year. The outcomes were hazards of first relapse, disability accumulation and disability improvement events. Sensitivity analyses were completed.

\section{Results}

The cohorts consisted of 37 (cladribine), 1940 (interferon), 1892

Abstract: (fingolimod) and 1410 patients (natalizumab). The probability of experiencing a relapse on cladribine was higher than on interferon $(p=0.05)$, similar to fingolimod $(p=0.31)$ and lower than on natalizumab $(p=0.042)$. The probability of disability accumulation on cladribine was similar to interferon $(p=0.37)$ and fingolimod $(p=0.089)$ but greater than natalizumab $(p=0.021)$. The probability of disability improvement was higher on cladribine than interferon $(p=0.00017)$, fingolimod $(p=0.0025)$ or natalizumab $(p=0.00099)$. Sensitivity analyses largely confirmed the above results.

\section{Conclusions}

Cladribine is an effective therapy for relapse-onset multiple sclerosis. Its effect on relapses is comparable to fingolimod and its effect on disability accrual is comparable to interferon $\beta$ and fingolimod. Cladribine may potentially associate with superior recovery from disability relative to interferon, fingolimod and natalizumab. 
Title

Cladribine versus fingolimod, natalizumab and interferon $\beta$ for multiple sclerosis

\section{Authors and affiliations}

Tomas Kalincik; CORe, Department of Medicine, University of Melbourne, Melbourne, Australia;

Department of Neurology, Royal Melbourne Hospital, Melbourne, Australia

Vilija Jokubaitis; Department of Medicine, University of Melbourne, Melbourne, Australia;

Department of Neurology, Royal Melbourne Hospital, Melbourne, Australia

Tim Spelman; Department of Medicine, University of Melbourne, Melbourne, Australia;

Department of Neurology, Royal Melbourne Hospital, Melbourne, Australia

Dana Horakova; Department of Neurology and Center of Clinical Neuroscience, General

University Hospital and Charles University in Prague, Prague, Czech Republic

Eva Havrdova; Department of Neurology and Center of Clinical Neuroscience, General

University Hospital and Charles University in Prague, Prague, Czech Republic

Maria Trojano; Department of Basic Medical Sciences, Neuroscience and Sense Organs,

University of Bari, Bari, Italy

Jeannette Lechner-Scott; University Newcastle, Newcastle, Australia

Alessandra Lugaresi; Department of Biomedical and Neuromotor Sciences, University of

Bologna, Bologna, Italy; IRCCS Istituto delle Scienze Neurologiche di Bologna, Bologna, Italy

Alexandre Prat; Hopital Notre Dame, Montreal, Canada; CHUM and Universite de Montreal,

Montreal, Canada

Marc Girard; Hopital Notre Dame, Montreal, Canada; CHUM and Universite de Montreal,

Montreal, Canada

Pierre Duquette; Hopital Notre Dame, Montreal, Canada; CHUM and Universite de Montreal,

Montreal, Canada

Pierre Grammond; CISSS Chaudière-Appalache, Levis, Canada

Claudio Solaro; Ospedale P. A. Micone, Genova, Italy

Francois Grand'Maison; Neuro Rive-Sud, Quebec, Canada

Raymond Hupperts; Zuyderland Ziekenhuis, Sittard, Netherlands

Julie Prevost; CSSS Saint-Jérôme, Saint-Jerome, Canada

Patrizia Sola; Nuovo Ospedale Civile Sant'Agostino/Estense, Modena, Italy

Diana Ferraro; Nuovo Ospedale Civile Sant'Agostino/Estense, Modena, Italy

Murat Terzi; Medical Faculty, 19 Mayis University, Samsun, Turkey

Ernest Butler; Monash Medical Centre, Melbourne, Australia

Mark Slee; Flinders University, Adelaide, Australia

Allan Kermode; Perron Institute, University of Western Australia, Perth, Australia; Institute of Immunology and Infectious Diseases, Murdoch University, Perth, Australia

Marzena Fabis-Pedrini; Perron Institute, University of Western Australia, Perth, Australia

Pamela McCombe; University of Queensland, Brisbane, Australia; Royal Brisbane and

Women's Hospital

Michael Barnett; Brain and Mind Centre, Sydney, Australia

Cameron Shaw; Geelong Hospital, Geelong, Australia

*Suzanne Hodgkinson; Liverpool Hospital, Sydney, Australia

*Helmut Butzkueven; Department of Medicine, University of Melbourne, Melbourne, Australia;

Department of Neurology, Royal Melbourne Hospital, Melbourne, Australia; Department of

Neurology, Box Hill Hospital, Monash University, Melbourne, Australia

on behalf of the MSBase Study Group

*equally contributing last authors

Keywords

cladribine, interferon, fingolimod, natalizumab, relapses, disability

\section{Corresponding author}

Tomas Kalincik, Level 4 Centre Royal Melbourne Hospital, 300 Grattan St, Melbourne VIC 3050; tel: +61 3 93424404; fax: +61 393495997

Word count - abstract: 197

Word count - manuscript: 3122 


\title{
Abstract
}

\section{Objective}

This propensity score-matched analysis from MSBase compared the effectiveness of cladribine with interferon $\beta$, fingolimod or natalizumab.

\begin{abstract}
Methods
We identified all patients with relapse-onset multiple sclerosis, exposure to the study therapies, and $\geq 1$-year on-treatment follow-up from MSBase. Three pairwise propensity score-matched analyses compared treatment outcomes over one year. The outcomes were hazards of first relapse, disability accumulation and disability improvement events. Sensitivity analyses were completed.
\end{abstract}

\section{Results}

The cohorts consisted of 37 (cladribine), 1940 (interferon), 1892 (fingolimod) and 1410 patients (natalizumab). The probability of experiencing a relapse on cladribine was higher than on interferon $(p=0.05)$, similar to fingolimod $(p=0.31)$ and lower than on natalizumab $(p=0.042)$. The probability of disability accumulation on cladribine was similar to interferon $(p=0.37)$ and fingolimod $(p=0.089)$ but greater than natalizumab $(p=0.021)$. The probability of disability improvement was higher on cladribine than interferon $(p=0.00017)$, fingolimod $(p=0.0025)$ or natalizumab $(p=0.00099)$. Sensitivity analyses largely confirmed the above results.

\section{Conclusions}

Cladribine is an effective therapy for relapse-onset multiple sclerosis. Its effect on relapses is comparable to fingolimod and its effect on disability accrual is comparable to interferon $\beta$ and fingolimod. Cladribine may potentially associate with superior recovery from disability relative to interferon, fingolimod and natalizumab. 


\section{Introduction}

Cladribine (2-chlorodeoxyadenosine) is a purinergic antimetabolite with a preferential effect on lymphocytes. Its intracellular metabolite, 2-chlorodeoxyadenosine triphosphate triggers lymphocyte apoptosis through inhibiting DNA synthesis and repair. ${ }^{1}$ Cladribine primarily triggers rapid and sustained reductions in numbers of CD4+ and CD20+ cells and transient reduction in numbers of CD8+ and natural killer cells. ${ }^{2}$ The compound has an established role as a treatment for several subtypes of leukaemia and Non-Hodgkin's lymphomas. It has recently been trialled as an immunotherapy for relapsing-remitting multiple sclerosis (MS). ${ }^{3}$

Superiority of cladribine to placebo in relapsing-remitting MS was demonstrated in the CLARITY phase 3 randomised controlled trial. ${ }^{4}$ Cladribine $3.5 \mathrm{mg} / \mathrm{kg}$ was superior to placebo in suppressing relapse activity (annualised relapse rate 0.14 vs. 0.33 , respectively) and increasing the probability of remaining relapse-free by $127 \%$. Cladribine was also associated with a relative $55 \%$ increase in the probability of remaining free from 3-month confirmed disability progression.

Cladribine was approved for indication relapsing-remitting MS in Australia in 2010, but the application was subsequently rejected in Europe and the US. At the present time, a resubmission to the European Medicines Agency is pending a decision. Evidence for effectiveness of cladribine compared to other agents is critical for determining its position in the current treatment landscape, however, no direct head-to-head comparisons of cladribine to other potent immunotherapies are available.

The Australian Cladribine Product Familiarisation Program in 2011 involved 144 patients with relapse-onset MS who received one course of cladribine in a clinical practice setting. ${ }^{5}$ Data from 90 of these patients, as well as additional 21 patients treated off-label, were captured in MSBase, a global observational cohort study of MS. MSBase has developed a track record of comparing effectiveness of MS immunotherapies using observational data. ${ }^{6,7}$ Here we conducted a propensity score-matched analysis of observational data from MSBase, including patients from Australian Cladribine Product Familiarisation Program, in order to compare the effectiveness of cladribine to that of three other common immunotherapies, interferon $\beta$, fingolimod and natalizumab. 


\section{Methods}

\section{Ethics Statement}

MSBase is an international observational cohort study of MS, registered with the World Health Organization International Clinical Trials Registry Platform, ID ACTRN12605000455662. ${ }^{8}$ The study was approved by the Melbourne Health Human Research Ethics Committee, and by the site institutional review boards (or exemptions were granted, according to local regulations). Written informed consent was obtained from enrolled patients, as required.

\section{Patients}

The inclusion criteria were definite relapse-onset $M S,{ }^{9,} 10$ exposure to one of the study therapies, $\geq 1$ year of continuous study monotherapy (in the cladribine group, patients were considered to be treated for 1 year after their exposure to cladribine), no prior exposure to alemtuzumab, mitoxantrone, rituximab or haematopoietic stem cell transplantation, minimum required recorded follow-up ( 3 months prior to treatment start and 2 disability scores $\geq 6$ months apart with at least one score recorded while on the study therapy) and minimum dataset (consisting of sex, age, date of first MS symptom, dates of clinical relapses, clinical MS course, and disability score at treatment commencement [-1 year to +1 month]).

\section{Procedures}

The included patients were treated with one of the study therapies: cladribine $(3.5 \mathrm{mg} / \mathrm{kg}$ total dose, initial treatment consisting of 2 courses completed in 2 weeks), interferon beta-1a ( $44 \mu \mathrm{g}$ s.c. three times weekly), fingolimod ( $0.5 \mathrm{mg}$ oral daily) and natalizumab (300 $\mu$ g i.v. every four weeks). Baseline was defined as the first commencement of the study therapy and patients were censored at discontinuing therapy, commencing a post-baseline disease modifying therapy, the last recorded disability score, or at the end of the first year post-baseline, whichever occurred first. 
The analysed data were recorded as part of routine clinical practice, mostly at tertiary MS centres, with data entry at the time of clinical visits. The MSBase Observational Plan stipulates minimum annual evaluations of neurological status of the included patients. Data entry portals were iMed or MSBase online data entry system. Rigorous automated quality assurance procedure was applied, assessing erroneous data entries, data density and generalisability as described elsewhere. ${ }^{11}$

\section{Outcomes}

The study endpoints were the proportion of patients free from relapses, disability accumulation events and disability improvement events while on study therapy.

A relapse was defined as new symptoms or exacerbation of existing symptoms persisting for $\geq 24$ hours, in the absence of concurrent illness/fever, and occurring $\geq 30$ days after a previous relapse. Information about relapses, their date of onset, symptomatology, severity, therapy and recovery was recorded by treated neurologists. Their confirmation with disability score was not required.

Disability was scored using Expanded Disability Status Scale (EDSS) by accredited EDSS scorers (Neurostatus certification was required at the participating centres), excluding the scores recorded within 30 days of a previous relapse. Disability accumulation was defined as an on-treatment increase in EDSS by 1 step ( 1.5 step if baseline EDSS was 0 and 0.5 steps if baseline EDSS was $>5.5$ ) confirmed by subsequent EDSS scores over $\geq 6$ months (irrespective of treatment status at confirmation). Disability improvement was defined as a decrease in EDSS by 1 step ( 1.5 step if baseline EDSS was 1.5 and 0.5 steps if baseline EDSS was $>6$; decrease from EDSS step 1 to step 0 was not be evaluated as confirmed disability improvement) confirmed by subsequent EDSS scores over $\geq 6$ months. ${ }^{12}$

\section{Statistical analysis}


Matching and statistical analyses were conducted using R (version 3.0.3), ${ }^{13}$ in three separate matched analyses of cladribine vs. interferon $\beta$, cladribine vs. fingolimod, or cladribine vs. natalizumab. Individual patients were matched on their propensity of receiving either of the compared therapies. ${ }^{6}{ }^{14}$ Individual propensity scores were calculated using a multivariable logistic improvement model of treatment allocation that used as independent variables the demographic and clinical variables available at the time of treatment assignation: sex, age, time from first MS symptom, EDSS, MS course, number of relapses in the prior 3 months, number of prior MS therapies, and the most effective prior MS therapy.

Patients were matched in a variable 10:1 ratio using nearest neighbour matching within a caliper of 0.5 standard deviations of the propensity score, without replacement and with exact match on EDSS (categorised as 2-EDSS step bins). All subsequent analyses were designed as paired models with weighting to adjust for the variable matching ratio. A maximum cumulative weight for each matched patient was 1 . The common on-treatment follow-up was determined in each matched pair as the shorter of the two patient follow-up periods (pairwise censoring) to mitigate attrition bias, informative censoring and the effect of differential treatment persistence. ${ }^{15}$ Tests of statistical inference were carried out at $\alpha=0.05$. No correction for false discovery rate was required as the number of tests of statistical inference per pairwise comparison was 9 . Cumulative hazards of freedom from relapses, EDSS accumulation and EDSS improvement were evaluated with weighted conditional proportional hazards models (Cox). Where the proportionality of hazards assumption was violated (as per Schoenfeld's global test), interaction term for treatment and time was included or Weibull models were used instead.

Robustness of the statistically significant differences to unidentified confounders was quantified with Rosenbaum sensitivity test for Hodges-Lehmann $\Gamma{ }^{16}$ Where no statistically significant differences were observed, analytical power was quantified as the minimum effect magnitude detectable within the available cohort at $1-\beta=0.8$ using 200 simulations.

Three sensitivity analyses were completed. The sensitivity analyses evaluated the robustness of the results to potential confounders, such as the matching parameters (using a loose definition of matching consisting of 20:1 variable matching within a large caliper of 5), matching exactly on 
MS course but not disability, and follow-up duration (allowing inclusion of patients with $<1$ year on-treatment follow-up).

\section{Results}

Of the 111 patients exposed to cladribine identified in the MSBase cohort, 90 patients took part in the Australian Cladribine Product Familiarisation Program and 21 were treated with cladribine off label (Supplementary Table S1). The numbers of eligible patients available for matching were 37 patients treated with cladribine, 1940 patients treated with interferon $\beta, 1892$ patients treated with fingolimod and 1410 patients treated with natalizumab (Figure 1). The 74 patients from the cladribine group who were excluded from the analysis had characteristics similar to the included cohort (mean age 45 years, disease duration 11 years, median EDSS 5, with 74\% patients diagnosed with relapsing-remitting MS and $12 \%$ with secondary progressive MS). The multivariable logistic regression models that were used to estimate the individual propensity scores indicated that patients treated with cladribine tended to be older than the patients treated with any of the three comparator therapies, with a greater disability than interferon $\beta$ and fingolimod, and with lower prior relapse activity than natalizumab (Supplementary Table S2). The numbers of patients retained in the pairwise-matched cohorts are shown in Table 1. The matching procedure decreased the between-group differences in propensity scores from 0.41 to 0.01 (by $97 \%$ ) for the comparison with interferon $\beta, 0.12$ to 0.001 (by $99 \%$ ) for the comparison with fingolimod, and from 0.30 to 0.01 (by $96 \%$ ) for the comparison of cladribine with natalizumab. Only mild to moderate differences in baseline characteristics were observed between the matched cohorts. On-treatment follow-up was identical between the matched cohorts as a result of pairwise censoring.

---insert Table 1 here---- 
The patients treated with cladribine $(n=22)$ were less likely to experience a relapse during the first year of treatment (hazard ratio, HR $0.6[95 \% \mathrm{Cl} 0.38-0.95], \mathrm{p}=0.050$ ) than the matched patients treated with interferon $\beta$ ( $n=167$; Figure 2). The proportions of relapse-free patients at the end of year one were $86 \%$ and $70 \%$, respectively. The probability of disability accumulation was similar in the two matched cohorts (HR 0.41 [95\% Cl 0.87-1.47], p=0.37). Patients treated with cladribine were relatively more likely to experience a disability improvement event during the first year on treatment (HR 15, [95\% Cl 3.6-59], $p=0.00017)$.

For both matched cladribine $(n=32)$ and fingolimod $(n=258)$ cohorts, the proportion of patients who remained relapse-free at the end of year one was $79 \%$ (Figure 3). Cumulative hazards of a relapse did not differ between the two groups (HR 1.2 [95\% Cl 0.83-1.8], $\mathrm{p}=0.31)$. The probability of disability accumulation was similar in the cladribine and fingolimod groups (HR 1.8 [95\% $\mathrm{Cl} 0.91-3.7], \mathrm{p}=0.089$ ). The probability of experiencing a disability improvement event was greater in the cladribine cohort when compared to the fingolimod cohort (HR $3.9[95 \% \mathrm{Cl} 1.6-$ 9.6], $P=0.0025)$.

The patients treated with cladribine $(n=26)$ were more likely to experience a relapse (HR 1.8 [95\% Cl 1.08-2.97], $p=0.042)$ than the matched patients treated with natalizumab $(n=174$; Figure 4). However, at the end of year one, the proportions of relapse-free patients were $80 \%$ and $81 \%$, respectively. The probability of disability accumulation was greater in the cladribine cohort (HR $2.5[95 \% \mathrm{Cl} 1.2-5.6], \mathrm{p}=0.021)$. The probability of experiencing a disability improvement event was greater among the patients treated with cladribine than those treated with natalizumab (HR 4 [95\% Cl 1.8-9.2], $\mathrm{p}=0.00099)$.

Sensitivity analyses largely confirmed the results of the primary analyses (Supplementary Table S3). The exception was the analysis the probability of a relapse, which did not replicate the difference between cladribine and interferon $\beta$ from the primary analysis, and the probability of a disability progression event, which showed a difference favouring cladribine over interferon $\beta$ in two sensitivity analyses. Also, the results of the sensitivity analysis using loose matching criteria showed results that differed from the primary analysis for the cladribine vs. fingolimod comparison. 
Analysis of the minimum detectable effect size was completed for each primary analysis that did not show significant difference between the matched groups (Supplementary Table S4). The analyses in which no statistically significant effect was observed were sufficiently powered to detect minimum differences of $62 \%$ for the probability of remaining relapse-free, and $2-42 \%$ for the probability of experiencing disability accumulation. According to the Rosenbaum sensitivity test for Hodges-Lehmann $\Gamma$, the comparisons of annual relapse rates were vulnerable to potential unmeasured confounders.

\section{Discussion}

In this observational, propensity score-matched study of patients with relapse-onset MS from MSBase, cladribine was superior to high-dose interferon $\beta$, similar to fingolimod and inferior to natalizumab in reducing relapse activity during the first year of treatment. The probability of confirmed disability accumulation during the first year on cladribine was greater than on natalizumab and was comparable to fingolimod and interferon $\beta$. Cladribine was more frequently associated with confirmed disability improvement than interferon $\beta$, fingolimod or natalizumab.

In a phase 3 placebo-controlled trial in relapsing-remitting MS (CLARITY), the two tested doses of cladribine $(3.5 \mathrm{mg} / \mathrm{kg}$ and $5.25 \mathrm{mg} / \mathrm{kg})$ were superior to placebo in reducing relapse activity and accumulation of disability. ${ }^{4}$ At 96 weeks, $80 \%$ of the cladribine $3.5 \mathrm{mg}$ group and $60 \%$ of the placebo group were free from relapses, and $91 \%$ of the cladribine $3.5 \mathrm{mg}$ group and $85 \%$ of the placebo group were free from 6-month confirmed increase in EDSS. ${ }^{17}$ Even though the followup in our study was shorter than in CLARITY, the proportions of patients free from relapses (80$90 \%)$ and 6-month confirmed disability progression (90\%) were in keeping with the data from the randomised trial. Two-year extension of the CLARITY trial (CLARITY-EXT) showed that in a majority of patients, the clinical benefits of cladribine used for 2 years were maintained for at least 4 years. ${ }^{18}$

A phase $2 \mathrm{~b}$ placebo-controlled trial of cladribine as an add-on to interferon $\beta$ (ONWARD) included $15 \%$ of patients with secondary progressive MS, a proportion that is similar to the representation of SPMS in our study. ${ }^{19}$ The study showed $63 \%$ reduction in the risk of a relapse 
in add-on cladribine when compared to placebo. A phase 3 placebo-controlled randomised trial (ORACLE MS) in clinically isolated syndrome showed that cladribine was associated with a $67 \%$ reduction in the risk of second relapse indicative of conversion to clinically definite $\mathrm{MS}^{20}$ In our study, the magnitude of the effect of cladribine on relapses was relatively lower, which could be explained by the comparison to active therapies.

Currently, there are no randomised or observational studies comparing the efficacy of cladribine to other disease modifying therapies. Therefore, the information that cladribine is superior to interferon $\beta$, similar to fingolimod and inferior to natalizumab in reducing relapse activity could help define the place of cladribine in the context of the other available MS therapies. With the exception of natalizumab, which was marginally superior in preventing disability accumulation during the first year on treatment, we were unable to demonstrate differences in disability accrual between cladribine and the comparator therapies. Finally, 6-month confirmed improvement of disability was observed in $10-20 \%$ of the cladribine cohort during the first year, which was superior to all three comparator therapies. This is of interest in the context of the comparison to natalizumab, which is known to be associated with a marked improvement in disability early after its commencement. ${ }^{6,7,21}$ It is also of note that, on average, the matched patients included in this study had relatively long-standing MS (median MS duration 10-15 years), with a median age of 44-50 years, were usually previously treated with other immunotherapies, and suffered from moderately severe disability (median EDSS scores of 3.54). Improvement in disability in a cohort with this profile is unexpected. Further validation of these results in patients with advanced MS is needed.

We conducted multiple steps to mitigate potential biases, including matching, pairwise censoring, and adjusting the statistical models, ${ }^{15}$ an approach mirroring our previous studies. ${ }^{6}$ A large number of patients treated with natalizumab, fingolimod, or interferon $\beta$ are available from the MSBase cohort; we were therefore able to achieve a satisfactory match on patients' demographic and clinical characteristics while maximising the power by one-to-multiple matching. Because the patients were exposed to only one dose of oral cladribine (i.e. two courses over two weeks) before the drug was withdrawn in Australia, we only analysed treatment outcomes during the initial year post-treatment. This study therefore did not evaluate 
treatment outcomes after a full dose of oral cladribine (i.e. after the second dose at 12 months). The primary analysis was conservative and limited to patients with a minimum 1-year follow-up. The main limitation of this study consists in the limited size of the cladribine cohort. Only a small number of patients with MS were exposed to cladribine world-wide, and the Australian Cladribine Product Familiarisation Program is, to our knowledge, the only available real-world MS cohort treated with oral cladribine. We maximised the use of this cohort by capturing, in MSBase, two thirds of the Australian treated population, of whom one quarter had sufficient longitudinal data to enable comparison of treatment outcomes, including relapse incidence, disability accumulation or disability improvement. While the power of this study is thus limited, it demonstrated a number of statistically significant differences between cladribine and the three comparator therapies. Where no difference was observed, the minimum detectable effect size was of moderate magnitude. In these instances, small or moderate differences in relapse frequency and the probability of disability accumulation or improvement would not have been detected. Another limitation is the restricted duration of the cladribine follow-up. We are therefore only able to comment on short-term outcomes on cladribine relative to the comparator therapies. While a good overall balance between the compared cohorts was achieved using propensity score matching, a potential residual imbalance was observed in some of the variables. In particular, patients treated with cladribine tended to be older and with longer time from MS onset than those treated with natalizumab (mean differences of 6 years and 4 years, respectively) and the cladribine cohort was enriched for patients with secondary progressive MS relative to the matched fingolimod cohort. These residual imbalances could potentially influence the outcomes of the comparative analyses, deflating relapse frequency and inflating disability accrual recorded in the cladribine cohorts when compared to the fingolimod and natalizumab cohorts. The absence of systematically captured safety data represents another limitation. Therefore, risk-benefit ratios of cladribine and the other therapies should be carefully considered by clinicians for each patient individually. Finally, the lack of MRI data prevented us from using MRI activity as a matching or an outcome variable.

The results presented were sensitive to hypothetical unmeasured confounders. To mitigate this risk, we demonstrated that the results were robust to variation in inclusion criteria and matching 
parameters. When using a loose match, thus simulating an unmatched scenario while preserving the paired structure of the models, the results differed from the primary analysis (namely for the cladribine vs. fingolimod comparison). This demonstrates the impact of stringent matching on the results of treatment comparisons, especially where the compared samples are heterogeneous.

In conclusion, cladribine is a proven effective therapy in relapse-onset multiple sclerosis. In our study, its effect on reducing relapse activity was comparable to fingolimod and its effect on disability accrual was comparable to interferon $\beta$ and fingolimod, while its association with sustained recovery from previously accrued disability was superior to interferon $\beta$, fingolimod and natalizumab. Currently, the submission of cladribine to the European Medicines Agency for relapsing-remitting MS is pending. Therefore, our comparative effectiveness results, while limited by the small cohort size, will help inform the role of cladribine in the management of MS. 


\section{Acknowledgements}

The MSBase Study contributors are listed in the Online Supplement.

\section{Study funding}

This study was financially supported by National Health and Medical Research Council of Australia [project grants 1129189 and 1083539 and practitioner fellowship 1080518]. The MSBase Foundation is a not-for profit organisation that receives support from Merck, Biogen, Novartis, Roche, Bayer Schering, Sanofi Genzyme, and Teva.

\section{Disclosure Statement}

Tomas Kalincik served on scientific advisory boards for Roche, Genzyme-Sanofi, Novartis, Merck and Biogen, received conference travel support and/or speaker honoraria from WebMD Global, Novartis, Biogen, Genzyme-Sanofi, Teva, BioCSL and Merck and has received research support from Biogen.

Vilija Jokubaitis received conference travel support from Novartis and Merck, and speaker honoraria from Biogen.

Tim Spelman received honoraria for consultancy, funding for travel and compensation for serving on scientific advisory boards from Biogen and speaker honoraria from Novartis.

Dana Horakova received speaker honoraria and consulting fees from Biogen, Merck, Teva and Novartis, as well as support for research activities from Biogen and research grants from Charles University in Prague (PRVOUK-P26/LF1/4 and Czech Ministry of Health (NT132374/2012).

Eva Havrdova received speaker honoraria and consultant fees from Actelion, Biogen, Merck, Novartis, Receptos, Genzyme and Teva, as well as support for research activities from Biogen, and Merck.

Helmut Butzkueven served on scientific advisory boards for Biogen, Novartis and SanofiAventis and has received conference travel support from Novartis, Biogen and Sanofi Aventis. He serves on steering committees for trials conducted by Biogen and Novartis, and has received research support from Merck, Novartis and Biogen.

Maria Trojano received speaker honoraria from Biogen-Idec, Bayer-Schering, Sanofi Aventis, Merck, Teva, Novartis and Almirall; has received research grants for her Institution from Biogen-Idec, Merck, and Novartis.

Jeannette Lechner-Scott accepted travel compensation from Novartis, Biogen and Merck. Her institution receives the honoraria for talks and advisory board commitment from Bayer Health Care, Biogen, Genzyme Sanofi, Merck, Novartis and Teva, has been involved in clinical trials with Biogen, Novartis and Teva.

Alessandra Lugaresi is a Bayer, Biogen, Genzyme, Merck Advisory Board Member. She received travel grants and honoraria from Bayer, Biogen, Merck, Novartis, Sanofi, Teva and Fondazione Italiana Sclerosi Multipla (FISM). Her institution received research grants from Bayer, Biogen, Merck, Novartis, Sanofi , Teva and Fondazione Italiana Sclerosi Multipla (FISM).

Alexandre Prat did not declare any competing interests.

Marc Girard received consulting fees from Teva Canada Innovation, Biogen, Novartis and Genzyme Sanofi; lecture payments from Teva Canada Innovation, Novartis and EMD . He has also received a research grant from Canadian Institutes of Health Research.

Pierre Duquette served on editorial boards and has been supported to attend meetings by EMD, Biogen, Novartis, Genzyme, and TEVA Neuroscience. He holds grants from the CIHR and the MS Society of Canada and has received funding for investigator-initiated trials from Biogen, Novartis, and Genzyme.

Pierre Grammond is a Novartis, Teva-neuroscience, Biogen and Genzyme advisory board member, consultant for Merck, received payments for lectures by Merck, TevaNeuroscience and Canadian Multiple sclerosis society, and received grants for travel from Teva-Neuroscience and Novartis.

Claudio Solaro did not declare any competing interests. 
Francois Grand'Maison received honoraria or research funding from Biogen, Genzyme, Novartis, Teva Neurosciences, Mitsubishi and ONO Pharmaceuticals.

Raymond Hupperts received honoraria as consultant on scientific advisory boards from Merck, Biogen, Genzyme-Sanofi and Teva, research funding from Merck and Biogen, and speaker honoraria from Sanofi-Genzyme and Novartis.

Julie Prevost accepted travel compensation from Novartis, Biogen, Genzyme, Teva, and speaking honoraria from Biogen, Novartis, Genzyme and Teva.

Patrizia Sola served on scientific advisory boards for Biogen Idec and TEVA, she has received funding for travel and speaker honoraria from Biogen Idec, Merck, Teva, Sanofi Genzyme, Novartis and Bayer and research grants for her Institution from Bayer, Biogen, Merck , Novartis, Sanofi, Teva.

Diana Ferraro received travel grants and/or speaker honoraria from Merck, TEVA, Novartis, Biogen and Sanofi-Genzyme.

Murat Terzi received travel grants from Merck, Novartis, Bayer-Schering, Merck and Teva; has participated in clinical trials by Sanofi Aventis, Roche and Novartis.

Ernest Butler did not declare any competing interests.

Mark Slee has participated in, but not received honoraria for, advisory board activity for Biogen, Merck, Bayer Schering, Sanofi Aventis and Novartis.

Allan Kermode received speaker honoraria and scientific advisory board fees from Bayer, BioCSL, Biogen, Genzyme, Innate Immunotherapeutics, Merck, Novartis, Sanofi, SanofiAventis, and Teva.

Pamela McCombe did not declare any competing interests.

Michael Barnett served on scientific advisory boards for Biogen, Novartis and Genzyme and has received conference travel support from Biogen and Novartis. He serves on steering committees for trials conducted by Novartis. His institution has received research support from Biogen, Merck and Novartis.

Cameron Shaw received travel assistance from Biogen and Novartis.

Suzanne Hodgkinson received honoraria and consulting fees from Novartis, Bayer Schering and Sanofi, and travel grants from Novartis, Biogen Idec and Bayer Schering.

Helmut Butzkueven served on scientific advisory boards for Biogen, Novartis and SanofiAventis and has received conference travel support from Novartis, Biogen and Sanofi Aventis. He serves on steering committees for trials conducted by Biogen and Novartis, and has received research support from Merck, Novartis and Biogen. 


\section{References}

1. Beutler E. Cladribine (2-chlorodeoxyadenosine). Lancet. 1992; 340: 952-6.

2. Juliusson G, Lenkei R and Liliemark J. Flow cytometry of blood and bone marrow cells from patients with hairy cell leukemia: phenotype of hairy cells and lymphocyte subsets after treatment with 2-chlorodeoxyadenosine. Blood. 1994; 83: 3672-81.

3. Rice GP, Filippi M and Comi G. Cladribine and progressive MS: clinical and MRI outcomes of a multicenter controlled trial. Cladribine MRI Study Group. Neurology. 2000; 54: 1145-55.

4. Giovannoni G, Comi G, Cook S, et al. A placebo-controlled trial of oral cladribine for relapsing multiple sclerosis. N Engl J Med. 2010; 362: 416-26.

5. Kalincik T, Lizak N, Jokubaitis V, et al. Australian cladribine experience. 32nd Conference of the European Committee for Treatment and Research in Multiple Sclerosis. London, UK2016.

6. Kalincik T, Horakova D, Spelman T, et al. Switch to natalizumab vs fingolimod in active relapsing-remitting multiple sclerosis. Ann Neurol. 2015; 77: 425-35.

7. Kalincik T, Brown JWL, Robertson N, et al. Comparison of alemtuzumab with natalizumab, fingolimod, and interferon beta for multiple sclerosis: a longitudinal study. The Lancet Neurology. 2017; 16: 271-81.

8. Butzkueven $\mathrm{H}$, Chapman J, Cristiano E, et al. MSBase: an international, online registry and platform for collaborative outcomes research in multiple sclerosis. Mult Scler. 2006; 12: 769-74.

9. Polman CH, Reingold SC, Edan G, et al. Diagnostic criteria for multiple sclerosis: 2005 revisions to the "McDonald Criteria". Ann Neurol. 2005; 58: 840-6.

10. Polman $\mathrm{CH}$, Reingold SC, Banwell $\mathrm{B}$, et al. Diagnostic criteria for multiple sclerosis: 2010 revisions to the McDonald criteria. Ann Neurol. 2011; 69: 292-302.

11. Kalincik T, Kuhle J, Pucci E, et al. Data quality evaluation for observational multiple sclerosis registries. Mult Scler. 2017; 23: 647-55.

12. Kalincik T, Cutter G, Spelman T, et al. Defining reliable disability outcomes in multiple sclerosis. Brain. 2015; 138: 3287-98.

13. R Development Core Team. R: A language and environment for statistical computing. Vienna, Austria: R Foundation for Statistical Computing, 2011.

14. Rosenbaum PR and Rubin DB. Reducing bias in observational studies using subclassification on the propensity score. Journal of American Statistical Association. 1984; 79: 516-24.

15. Kalincik T and Butzkueven H. Observational data: Understanding the real MS world. Mult Scler. 2016; 22: 1642-8.

16. Rosenbaum PR. Observational studies. 2nd ed. New York, NY: Springer-Verlag, 2002. 17. Giovannoni G, Cook S, Rammohan K, et al. Sustained disease-activity-free status in patients with relapsing-remitting multiple sclerosis treated with cladribine tablets in the CLARITY study: a post-hoc and subgroup analysis. Lancet Neurol. 2011; 10: 329-37.

18. Giovannoni G, Comi G, Cook S, et al. Clinical Efficacy of Cladribine Tablets in Patients with Relapsing-Remitting Multiple Sclerosis (RRMS): Final Results from the 120-Week Phase IIIb Extension Trial to the CLARITY Study (P3.028). Neurology. 2016; 86: P3.028.

19. Montalban X, Cohen B, Leist T, Moses H, Hicking C and Dangond F. Efficacy of Cladribine Tablets as Add-On to IFN-beta Therapy in Patients with Active Relapsing MS: Final Results from the Phase II ONWARD Study. Neurology. 2016; 86: P3.029.

20. Leist TP, Comi G, Cree BA, et al. Effect of oral cladribine on time to conversion to clinically definite multiple sclerosis in patients with a first demyelinating event (ORACLE MS): a phase 3 randomised trial. Lancet Neurol. 2014; 13: 257-67.

21. Belachew S, Phan Ba R, Bartholome E, et al. Natalizumab induces a rapid improvement of disability status and ambulation after failure of previous therapy in relapsingremitting multiple sclerosis. Eur J Neurol. 2011; 18: 240-5. 
Table 1

Baseline characteristics of matched patient groups

\begin{tabular}{|c|c|c|c|c|c|c|c|c|c|}
\hline & $\begin{array}{l}\text { Cladribine } \\
(n=22)\end{array}$ & $\begin{array}{l}\text { Interferon } \beta \\
(\mathrm{n}=167)\end{array}$ & d & $\begin{array}{l}\text { Cladribine } \\
(n=32)\end{array}$ & $\begin{array}{l}\text { Fingolimod } \\
(n=258)\end{array}$ & d & $\begin{array}{l}\text { Cladribine } \\
(n=26)\end{array}$ & $\begin{array}{l}\text { Natalizumab } \\
(\mathrm{n}=174)\end{array}$ & d \\
\hline female, nr (\%) & $17(77 \%)$ & $125(75 \%)$ & & $26(81 \%)$ & $201(78 \%)$ & & $20(77 \%)$ & $125(72 \%)$ & \\
\hline age, yr, mean \pm SD & $49 \pm 10$ & $45 \pm 8$ & 0.43 & $50 \pm 10$ & $48 \pm 8$ & 0.24 & $50 \pm 9$ & $44 \pm 10$ & 0.57 \\
\hline $\begin{array}{l}\text { disease duration, yr, median } \\
\text { (quartiles) }\end{array}$ & $13.5(5.6-18.3)$ & $11.2(6.5-17.1)$ & 0.21 & $14.1(7.6-23.8)$ & $13.8(8-19.5)$ & 0.20 & $14(6.2-17.9)$ & $10(4.5-17.2)$ & 0.34 \\
\hline $\begin{array}{l}\text { disability, EDSS, median } \\
\text { (quartiles) }\end{array}$ & $3.75(2.25-5.9)$ & $3.5(2-4.5)$ & 0.21 & $4.5(3-6)$ & $3.5(2-5.5)$ & 0.20 & $4(2-6)$ & $3.5(2-5.5)$ & 0.34 \\
\hline \multicolumn{10}{|l|}{ disease course, patients } \\
\hline relapsing-remitting & $19(86 \%)$ & $151(90 \%)$ & & $24(75 \%)$ & $216(84 \%)$ & & $22(85 \%)$ & $154(89 \%)$ & \\
\hline secondary progressive & $3(14 \%)$ & $16(10 \%)$ & & $8(25 \%)$ & $42(16 \%)$ & & $4(15 \%)$ & $20(11 \%)$ & \\
\hline $\begin{array}{l}\text { relapses } 3 \text { months pre-baseline, } \\
\text { mean } \pm S D\end{array}$ & $0.1 \pm 0.4$ & $0.2 \pm 0.4$ & 0.23 & $0.2 \pm 0.4$ & $0.1 \pm 0.4$ & 0.07 & $0.1 \pm 0.3$ & $0.2 \pm 0.4$ & 0.27 \\
\hline $\begin{array}{l}\text { visit interval, months, median } \\
\text { (quartiles) }\end{array}$ & $6(4-12)$ & $6(3-6)$ & 0.68 & $6(4-12)$ & $6(3-6)$ & 0.24 & $6(4-12)$ & $4(3-6)$ & 0.47 \\
\hline $\begin{array}{l}\text { previous therapies, nr, median } \\
\text { (quartiles) }\end{array}$ & $1(1-2)$ & $1(1-2)$ & 0.29 & $2(1-3)$ & $2(1-3)$ & 0.01 & $1(1-3)$ & $1(1-2)$ & 0.05 \\
\hline \multicolumn{10}{|l|}{$\begin{array}{l}\text { most active previous therapy, } \\
\text { patients }\end{array}$} \\
\hline IFN/GA & $20(91 \%)$ & $150(90 \%)$ & & $23(72 \%)$ & $187(72 \%)$ & & $24(92 \%)$ & $155(89 \%)$ & \\
\hline Dimethyl fumarate & $0(0 \%)$ & $0(0 \%)$ & & $1(3 \%)$ & $5(2 \%)$ & & $1(4 \%)$ & $3(2 \%)$ & \\
\hline Fingolimod & $0(0 \%)$ & $1(1 \%)$ & & $0(0 \%)$ & $0(0 \%)$ & & $1(4 \%)$ & $10(6 \%)$ & \\
\hline Natalizumab & $1(5 \%)$ & $4(2 \%)$ & & $7(22 \%)$ & $60(23 \%)$ & & $0(0 \%)$ & $0(0 \%)$ & \\
\hline none & $1(5 \%)$ & $12(7 \%)$ & & $1(3 \%)$ & $6(2 \%)$ & & $0(0 \%)$ & $6(3 \%)$ & \\
\hline
\end{tabular}




\section{Figure legends}

Figure 1

Patient disposition

CIS, clinically isolated syndrome; EDSS, Expanded Disability Status Scale; MS, multiple sclerosis; PPMS, primary progressive multiple sclerosis

\section{Figure 2}

Probability of first relapse (A), disability accumulation (B), disability improvement (C) events and disability in EDSS [median $\pm 95 \%$ confidence interval] (D) in matched patients treated with cladribine or interferon $\beta$.

95\% Cl, 95\% confidence interval; EDSS, Expanded Disability Status Scale

\section{Figure 3}

Probability of first relapse $(A)$, disability accumulation (B), disability improvement $(C)$ events and disability in EDSS [median $\pm 95 \%$ confidence interval] (D) in matched patients treated with cladribine or fingolimod.

95\% Cl, 95\% confidence interval; EDSS, Expanded Disability Status Scale

\section{Figure 4}

Probability of first relapse (A), disability accumulation (B), disability improvement (C) events and disability in EDSS [median $\pm 95 \%$ confidence interval] (D) in matched patients treated with cladribine or natalizumab.

95\% Cl, 95\% confidence interval; EDSS, Expanded Disability Status Scale 
Patient disposition

CIS, clinically isolated syndrome; EDSS, Expanded Disability Status Scale; MS, multiple sclerosis; PPMS, primary progressive multiple sclerosis

$199 \times 54 \mathrm{~mm}(300 \times 300 \mathrm{DPI})$ 
A

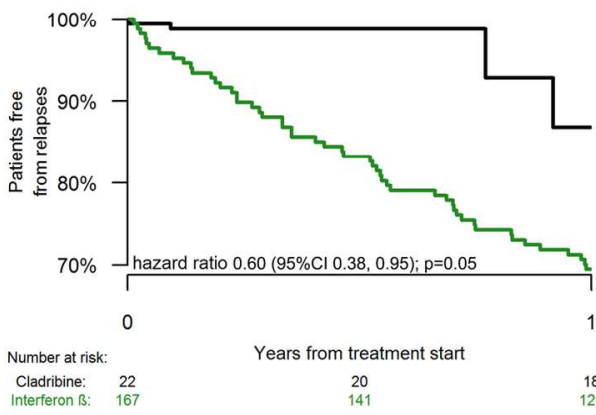

C

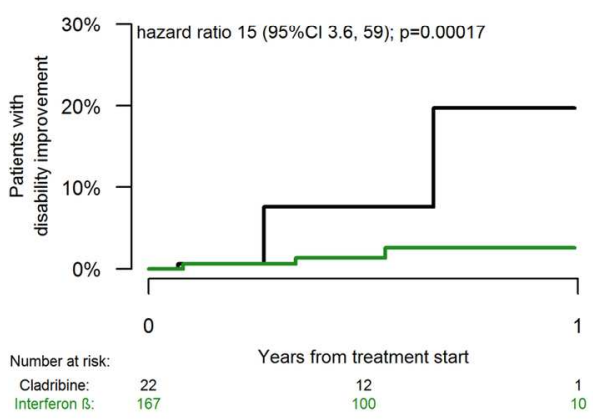

B

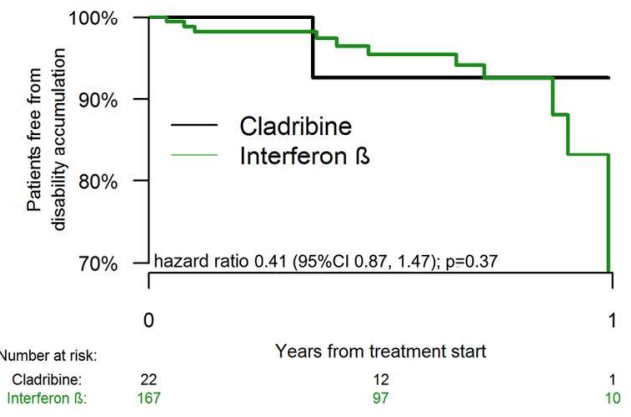

D

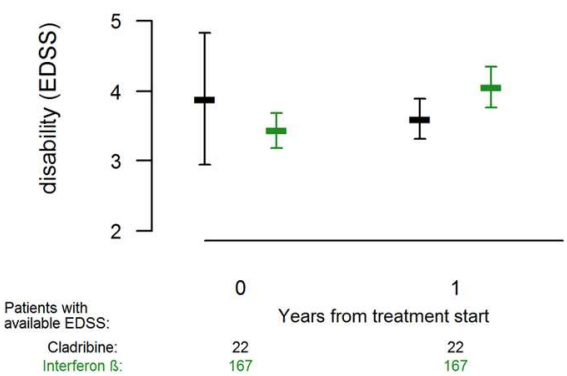

Probability of first relapse (A), disability accumulation (B), disability improvement (C) events and disability in EDSS [median $\pm 95 \%$ confidence interval] (D) in matched patients treated with cladribine or interferon $\beta$. 95\%CI, 95\% confidence interval; EDSS, Expanded Disability Status Scale

$149 \times 124 \mathrm{~mm}(300 \times 300 \mathrm{DPI})$ 
A

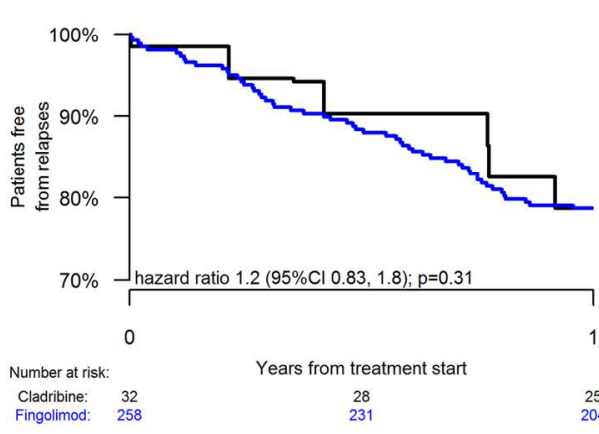

C

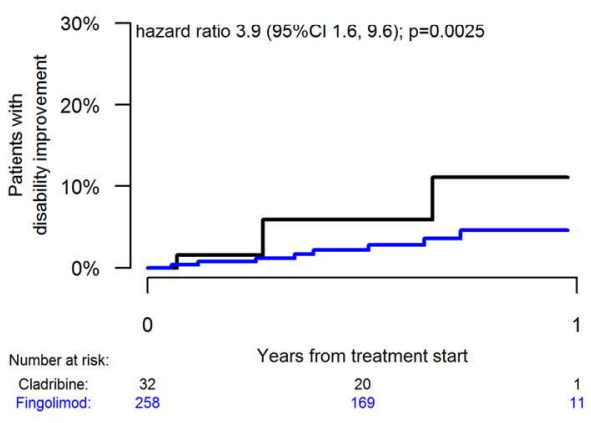

B

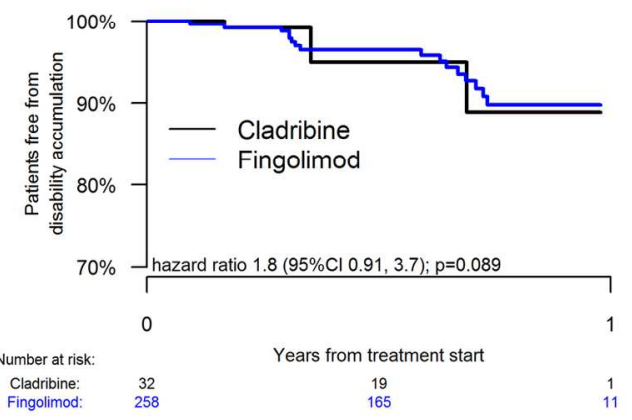

D

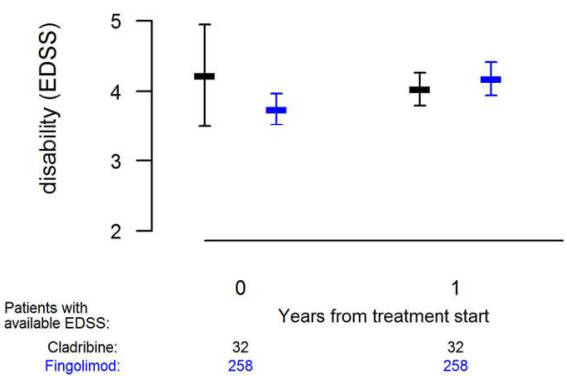

Probability of first relapse (A), disability accumulation (B), disability improvement (C) events and disability in EDSS [median $\pm 95 \%$ confidence interval] $(D)$ in matched patients treated with cladribine or fingolimod. 95\%CI, 95\% confidence interval; EDSS, Expanded Disability Status Scale

$149 \times 124 \mathrm{~mm}(300 \times 300 \mathrm{DPI})$ 
A B
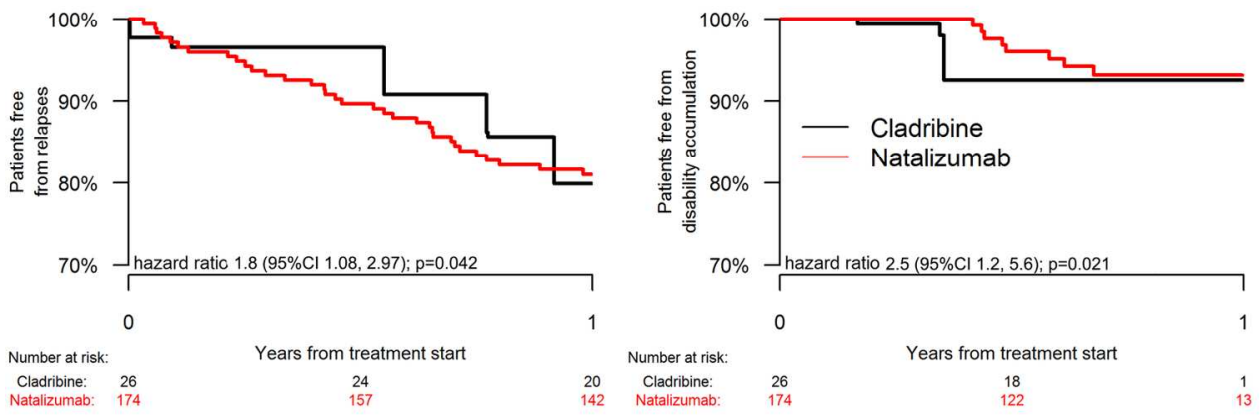

C

D
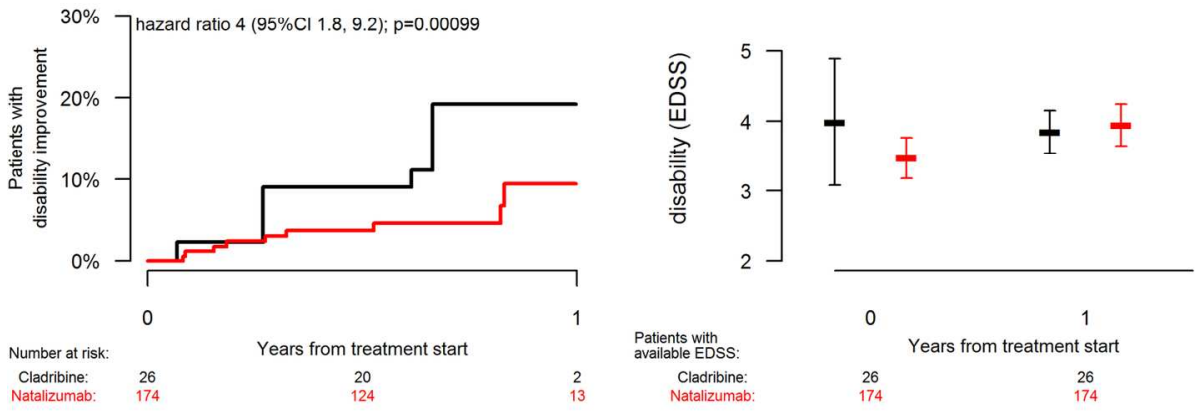

Probability of first relapse (A), disability accumulation (B), disability improvement (C) events and disability in EDSS [median $\pm 95 \%$ confidence interval] (D) in matched patients treated with cladribine or natalizumab. 95\%CI, 95\% confidence interval; EDSS, Expanded Disability Status Scale

$149 \times 124 \mathrm{~mm}(300 \times 300 \mathrm{DPI})$ 


\section{List of Contributors}

The following contributors participated in data acquisition:

From CIREN, Havana, Cuba, Dr Jose Antonio Cabrera-Gomez.

From MS Clinic, Hopital Tenon, Paris, France, Dr Etienne Roullet.

From University Hospital Nijmegen, Nijmegen, Netherlands, Dr Cees Zwanikken.

From Francicus Ziekenhuis, Roosendaal, Netherlands, Dr Leontien Den braber-Moerland.

From Hospital Fernandez, Capital Federal, Argentina, Dr Norma Deri.

From Institute of Neuroscience Buenos Aires, Buenos Aires, Argentina, Dr Maria Laura Saladino.

From Westmead Hospital, Sydney, Australia, Dr Steve Vucic.

From Royal Hobart Hospital, Hobart, Australia, Dr Bruce Taylor.

From The Alfred, Melbourne, Australia, Dr Olga Skibina.

From Austin Health, Melbourne, Australia, Dr Richard Macdonell.

From St. Vincent's Hospital, Australia, Dr Neil Shuey.

From Cliniques Universitaires Saint-Luc, Brussels, Belgium, Dr Vincent Van Pesch.

From Universidade Metropolitana de Santos, Santos, Brazil, Dr Yara Fragoso.

From Jewish General Hospital, Montreal, Canada, Dr Fraser Moore.

From Kommunehospitalet, Arhus C, Denmark, Dr Thor Petersen.

From Hospital Universitario Virgen de Valme, Seville, Spain, Dr Guillermo Izquierdo, Dr Ricardo Fernandez Bolaños.

From Hospital Universitario Donostia, San Sebastián, Spain, Dr Javier Olascoaga.

From Hospital Clinico San Carlos, Madrid, Spain, Dr Celia Oreja-Guevara.

From Hospital de Galdakao-Usansolo, Galdakao, Spain, Dr Jose Luis Sanchez-Menoyo.

From Hospital Germans Trias i Pujol, Badalona, Spain, Dr Cristina Ramo.

From Hosptal Universitario de la Ribera, Alzira, Spain, Dr Jose Andres Dominguez.

From Jahn Ferenc Teaching Hospital, Budapest, Hungary, Dr Csilla Rozsa, Dr Krisztian Kasa.

From University of Debrecen, Debrecen, Hungary, Dr Tunde Csepany.

From Péterfy Sandor Hospital, Budapest, Hungary, Dr Krisztina Kovacs.

From Szent Imre Hospital, Budapest, Hungary, Dr Eniko Dobos.

From Assaf Harofeh Medical Center, Beer-Yaakov, Israel, Dr Shlomo Flechter.

From Isfahan University of Medical Sciences, Isfahan, Iran, Dr Vahid Shaygannejad.

From Azienda Sanitaria Unica Regionale Marche - AV3, Macerata, Italy, Dr Eugenio Pucci, Dr Matteo Diamanti, Dr Elisabetta Cartechini.

From University of Florence, Florence, Italy, Dr Maria Pia Amato.

From C. Mondino National Neurological Institute, Pavia, Italy, Dr Roberto Bergamaschi.

From Ospedali Riuniti di Salerno, Salerno, Italy, Dr Gerardo Iuliano.

From University of Parma, Parma, Italy, Dr Franco Granella.

From Azienda Ospedaliera di Rilievo Nazionale San Giuseppe Moscati Avellino, Avellino, Italy, Dr Daniele Spitaleri.

From Amiri Hospital, Kuwait City, Kuwait, Dr Raed Alroughani.

From Groene Hart Ziekenhuis, Gouda, Netherlands, Dr Freek Verheul.

From Royal Hospital, Muscat, Oman, Dr Jabir Alkhaboori.

From Hospital São João, Porto, Portugal, Dr Maria Edite Rio.

From Central Military Emergency University Hospital, Bucharest, Romania, Dr Carmen-Adella Sirbu.

From King Fahad Specialist Hospital-Dammam, Khobar, Saudi Arabia, Dr Talal Al-Harbi.

From KTU Medical Faculty Farabi Hospital, Trabzon, Turkey, Dr Cavit Boz.

From Istanbul University Cerrahpasa School of Medicine, Istanbul, Turkey, Dr Ayse Altintas.

From Box Hill Hospital, Melbourne, Australia, Ms Jodi Haartsen.

From University of Melbourne, Melbourne, Australia, Dr Anneke van der Walt, Dr Mark Marriott, Dr Trevor Kilpatrick, Dr John King, Dr Katherine Buzzard, Dr Ai-Lan Nguyen, Dr Chris Dwyer, Dr Mastura Monif.

From University “G. d’Annunzio”, Italy, Dr Giovanna De Luca, Dr Valeria Di Tommaso, Dr Daniela Travaglini, Dr Erika Pietrolongo, Dr Maria di Ioia, Dr Deborah Farina, Dr Luca Mancinelli.

From Nuovo Ospedale Civile Sant'Agostino/Estense, Modena, Italy, Dr Francesca Vitetta, Dr Anna Maria Simone.

\section{Administrative and technical support was provided by:}

From the MSBase Administrations Ms Charlotte Sartori and Ms Sabah Quddus.

From Rodanotech, Geneva, Switzerland; Mr Samir Mechati, Mr Matthieu Corageoud, Mr Alexandre Bulla. 


\section{Supplementary Table S1 \\ Patient disposition per centre and therapy}

Patients

Hospital Fernandez, Capital Federal, Argentina

Hospital Italiano, Buenos Aires, Argentina Sir Charles Gairdner Hospital, Nedlands, Australia

Brain and Mind Centre, Sydney, Australia University of Melbourne, Melbourne, Australia

University Newcastle, Newcastle,

Australia

Geelong Hospital, Geelong, Australia

St Vincents Hospital, Fitzroy, Melbourne, Australia

Monash Medical Centre, Melbourne, Australia

Liverpool Hospital, Sydney, Australia

Box Hill Hospital, Melbourne, Australia Westmead Hospital, Sydney, Australia Flinders University, Adelaide, Australia University of Queensland, Brisbane, Australia

Royal Hobart Hospital, Hobart, Australia The Alfred, Melbourne, Australia Austin Health, Melbourne, Australia Cliniques Universitaires Saint-Luc, Brussels, Belgium

Universidade Metropolitana de Santos, Santos, Brazil

CSSS Saint-Jérôme, Saint-Jerome, Canada

Jewish General Hospital, Montreal, Canada

Hopital Notre Dame, Montreal, Canada CISSS Chaudière-Appalache, Levis, Canada

Neuro Rive-Sud, Quebec, Canada General University Hospital and Charles University in Prague, Prague, Czech Republic

Kommunehospitalet, Arhus C, Denmark Hospital Universitario Virgen de Valme, Seville, Spain

Hospital Universitario Donostia, San Sebastián, Spain

Hospital Clinico San Carlos, Madrid, Spain

Hospital Universitario Virgen Macarena, Sevilla, Spain

Hospital de Galdakao-Usansolo, Galdakao, Spain

Hospital Germans Trias i Pujol, Badalona, Spain

- total

Cladribine Interferon $\boldsymbol{\beta}$ Fingolimod Natalizumab

$\begin{array}{rrrrr}1 & 0 & 0 & 0 & 1 \\ 2 & 0 & 1 & 1 & 0 \\ 3 & 3 & 0 & 0 & 0 \\ 2 & 1 & 0 & 1 & 0 \\ 39 & 2 & 3 & 20 & 14\end{array}$

25

$$
0
$$

4 
Patients - total Cladribine Interferon $\beta$ Fingolimod Natalizumab

Hospital Universitario de la Ribera, Alzira, Spain

South East Trust, Belfast, United Kingdom

Veszprém Megyei Csolnoky Ferenc Kórház zrt., Veszprem, Hungary Jahn Ferenc Teaching Hospital, Budapest, Hungary University of Debrecen, Debrecen, Hungary

Szent Imre Hospital, Budapest, Hungary Assaf Harofeh Medical Center, BeerYaakov, Israel University "G. d'Annunzio ", Chieti, Italy Azienda Sanitaria Unica Regionale Marche - AV3, Macerata, Italy University of Bari, Bari, Italy University of Florence, Florence, Italy C. Mondino National Neurological Institute, Pavia, Italy

Ospedali Riuniti di Salerno, Salerno, Italy University of Parma, Parma, Italy Azienda Ospedaliera di Rilievo Nazionale San Giuseppe Moscati Avellino, Avellino, Italy

Nuovo Ospedale Civile

Sant'Agostino/Estense, Modena, Italy Ospedale P. A. Micone, Genova, Italy Amiri Hospital, Kuwait City, Kuwait University Hospital Nijmegen, Nijmegen, Netherlands

Zuyderland Ziekenhuis, Sittard, Netherlands

Jeroen Bosch Ziekenhuis, Den Bosch, Netherlands

Groene Hart Ziekenhuis, Gouda, Netherlands

Hospital São João, Porto, Portugal KTU Medical Faculty Farabi Hospital, Trabzon, Turkey 19 Mayis University, Samsun, Turkey

1

1

1

1

2

10

Interferon $\beta$

1

1

0

1

2

2

1

0

7

1

9

0

2

0

3

3

3

12

2

0

9

1

3

2

2

0 
39

40

41

42 no minimum follow-up

43 exact match on MS course

44 loose matching criteria

45 ladribine vs. fingolimod 47 primary analysis

48 no minimum follow-up

49 exact match on MS course

50 loose matching criteria

51 दो: 53 primary analysis

54 no minimum follow-up

55 exact match on MS course

57 loose matching criteria

\begin{tabular}{|c|c|c|c|c|c|c|}
\hline & \multicolumn{2}{|c|}{ interferon $\beta$} & \multicolumn{2}{|c|}{ fingolimod } & \multicolumn{2}{|c|}{ natalizumab } \\
\hline & coefficient & p value & coefficient & p value & coefficient & p value \\
\hline sex & & n.s. & & n.s. & & n.s. \\
\hline age, year & -0.13 & $10^{-6}$ & -0.10 & $10^{-5}$ & -0.13 & $10^{-7}$ \\
\hline disease duration & & n.s. & & n.s. & & n.s. \\
\hline disability, EDSS step & -0.32 & 0.045 & -0.30 & 0.014 & & n.s. \\
\hline relapses 3 months pre-baseline & & n.s. & & n.s. & 1.61 & 0.28 \\
\hline disease course & & n.s. & & n.s. & & n.s. \\
\hline prior therapies, $\mathrm{nr}$ & -1.01 & 0.0003 & & n.s. & & n.s. \\
\hline most active prior therapy & & & & n.s. & & \\
\hline fingolimod & -4.35 & 0.011 & & n.s. & & n.s. \\
\hline interferon beta / glatiramer acetate & & n.s. & & n.s. & & n.s. \\
\hline natalizumab & -4.52 & 0.002 & & n.s. & & n.s. \\
\hline dimethyl fumarate & & n.s. & & n.s. & & n.s. \\
\hline teriflunomide & & - & & n.s. & & n.s. \\
\hline
\end{tabular}

\section{Supplementary Table S2}

Multivariable logistic regression models used to calculate propensity scores

reference: cladribine; n.s., no evidence for the association of independent variable with treatment allocation $(p>0.05)$

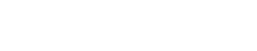




\section{Supplementary Table S4}

Results of the power analyses

analysis

cladribine vs. interferon $\beta$

cladribine vs. fingolimod

cladribine vs. natalizumab outcomes

\begin{tabular}{|c|c|c|}
\hline $\begin{array}{l}\text { probability } \\
\text { of a } \\
\text { relapse }\end{array}$ & $\begin{array}{l}\text { disability } \\
\text { accumulation } \\
\text { hazard }\end{array}$ & $\begin{array}{l}\text { disability } \\
\text { improvement } \\
\text { probability }\end{array}$ \\
\hline
\end{tabular}

The table shows minimum detectable differences for the disease outcomes whose analyses did not reach the level of statistical significance in the primary analyses. The differences are shown as proportion of the cumulative hazards. 
STROBE Statement - checklist of items that should be included in reports of observational studies

Title: Cladribine versus fingolimod, natalizumab and interferon $\beta$ for multiple sclerosis

\begin{tabular}{|c|c|c|c|}
\hline & $\begin{array}{l}\text { Item } \\
\text { No. }\end{array}$ & Recommendation & $\begin{array}{l}\text { Page } \\
\text { No. }\end{array}$ \\
\hline \multirow[t]{2}{*}{ Title and abstract } & \multirow[t]{2}{*}{1} & (a) Indicate the study's design with a commonly used term in the title or the abstract & 2 \\
\hline & & $\begin{array}{l}\text { (b) Provide in the abstract an informative and balanced summary of what was done and what was } \\
\text { found }\end{array}$ & 2 \\
\hline \multicolumn{4}{|l|}{ Introduction } \\
\hline Background/rationale & 2 & Explain the scientific background and rationale for the investigation being reported & 3 \\
\hline Objectives & 3 & State specific objectives, including any prespecified hypotheses & 3 \\
\hline \multicolumn{4}{|l|}{ Methods } \\
\hline Study design & 4 & Present key elements of study design early in the paper & $3-4$ \\
\hline Setting & 5 & $\begin{array}{l}\text { Describe the setting, locations, and relevant dates, including periods of recruitment, exposure, } \\
\text { follow-up, and data collection }\end{array}$ & 3 \\
\hline \multirow[t]{2}{*}{ Participants } & \multirow[t]{2}{*}{6} & $\begin{array}{l}\text { (a) Cohort study-Give the eligibility criteria, and the sources and methods of selection of } \\
\text { participants. Describe methods of follow-up } \\
\text { Case-control study-Give the eligibility criteria, and the sources and methods of case } \\
\text { ascertainment and control selection. Give the rationale for the choice of cases and controls } \\
\text { Cross-sectional study - Give the eligibility criteria, and the sources and methods of selection of } \\
\text { participants }\end{array}$ & 3 \\
\hline & & $\begin{array}{l}\text { (b) Cohort study_-For matched studies, give matching criteria and number of exposed and } \\
\text { unexposed } \\
\text { Case-control study_-For matched studies, give matching criteria and the number of controls per } \\
\text { case }\end{array}$ & 4 \\
\hline Variables & 7 & $\begin{array}{l}\text { Clearly define all outcomes, exposures, predictors, potential confounders, and effect modifiers. } \\
\text { Give diagnostic criteria, if applicable }\end{array}$ & $3-4$ \\
\hline $\begin{array}{l}\text { Data sources/ } \\
\text { measurement }\end{array}$ & $8^{*}$ & $\begin{array}{l}\text { For each variable of interest, give sources of data and details of methods of assessment } \\
\text { (measurement). Describe comparability of assessment methods if there is more than one group }\end{array}$ & $3-4$ \\
\hline Bias & 9 & Describe any efforts to address potential sources of bias & $4-5$ \\
\hline Study size & 10 & Explain how the study size was arrived at & $3-4$ \\
\hline
\end{tabular}

Continued on next page 


\begin{tabular}{|c|c|c|c|}
\hline $\begin{array}{l}\text { Quantitative } \\
\text { variables }\end{array}$ & 11 & $\begin{array}{l}\text { Explain how quantitative variables were handled in the analyses. If applicable, describe which } \\
\text { groupings were chosen and why }\end{array}$ & $4-5$ \\
\hline \multirow{7}{*}{$\begin{array}{l}\text { Statistical } \\
\text { methods }\end{array}$} & \multirow[t]{7}{*}{12} & (a) Describe all statistical methods, including those used to control for confounding & $4-5$ \\
\hline & & (b) Describe any methods used to examine subgroups and interactions & $4-5$ \\
\hline & & (c) Explain how missing data were addressed & $3-5$ \\
\hline & & (d) Cohort study —If applicable, explain how loss to follow-up was addressed & 4 \\
\hline & & Case-control study —-If applicable, explain how matching of cases and controls was addressed & \\
\hline & & $\begin{array}{l}\text { Cross-sectional study - If applicable, describe analytical methods taking account of sampling } \\
\text { strategy }\end{array}$ & \\
\hline & & (e) Describe any sensitivity analyses & \\
\hline Results & & 更 & \\
\hline \multirow[t]{3}{*}{ Participants } & \multirow[t]{3}{*}{$13 *$} & $\begin{array}{l}\text { (a) Report numbers of individuals at each stage of study—eg numbers potentially eligible, examined } \\
\text { for eligibility, confirmed eligible, included in the study, completing follow-up, and analysed }\end{array}$ & 5 \\
\hline & & (b) Give reasons for non-participation at each stage & Fig 1 \\
\hline & & (c) Consider use of a flow diagram & Fig 1 \\
\hline \multirow[t]{3}{*}{ Descriptive data } & \multirow[t]{3}{*}{$14 *$} & $\begin{array}{l}\text { (a) Give characteristics of study participants (eg demographic, clinical, social) and information on } \\
\text { exposures and potential confounders }\end{array}$ & Tab 1 \\
\hline & & (b) Indicate number of participants with missing data for each variable of interest & Fig 1 \\
\hline & & (c) Cohort study—Summarise follow-up time (eg, average and total amount) & Tab 1 \\
\hline \multirow[t]{3}{*}{ Outcome data } & \multirow[t]{3}{*}{$15^{*}$} & Cohort study-Report numbers of outcome events or summary measures over time & $5-6$ \\
\hline & & Case-control study-Report numbers in each exposure category, or summary measures of exposure & \\
\hline & & Cross-sectional study—Report numbers of outcome events or summary measures & \\
\hline \multirow[t]{3}{*}{ Main results } & \multirow[t]{3}{*}{16} & $\begin{array}{l}\text { (a) Give unadjusted estimates and, if applicable, confounder-adjusted estimates and their precision } \\
\text { (eg, } 95 \% \text { confidence interval). Make clear which confounders were adjusted for and why they were } \\
\text { included }\end{array}$ & 5-6, Fig 2-4 \\
\hline & & (b) Report category boundaries when continuous variables were categorized & $\mathrm{n} / \mathrm{a}$ \\
\hline & & $\begin{array}{l}\text { (c) If relevant, consider translating estimates of relative risk into absolute risk for a meaningful time } \\
\text { period }\end{array}$ & $\mathrm{n} / \mathrm{a}$ \\
\hline
\end{tabular}

Continued on next page 
Discussion

Key results

18 Summarise key results with reference to study objectives

6

Limitations

19 Discuss limitations of the study, taking into account sources of potential bias or imprecision. Discuss

7 both direction and magnitude of any potential bias

Interpretation 20 Give a cautious overall interpretation of results considering objectives, limitations, multiplicity of analyses, results from similar studies, and other relevant evidence

\begin{tabular}{|c|c|c|c|}
\hline Generalisability & 21 & Discuss the generalisability (external validity) of the study results & $6-7$ \\
\hline \multicolumn{4}{|c|}{ Other information } \\
\hline Funding & 22 & $\begin{array}{l}\text { Give the source of funding and the role of the funders for the present study and, if applicable, for the } \\
\text { original study on which the present article is based }\end{array}$ & 9 \\
\hline
\end{tabular}

*Give information separately for cases and controls in case-control studies and, if applicable, for exposed and unexposed groups in cohort and cross-sectional studies.

Note: An Explanation and Elaboration article discusses each checklist item and gives methodological background and published examples of transparent reporting. The STROBE checklist is best used in conjunction with this article (freely available on the Web sites of PLoS Medicine at http://www.plosmedicine.org/, Annals of Internal Medicine at http://www.annals.org/, and Epidemiology at http://www.epidem.com/). Information on the STROBE Initiative is available at www.strobe-statement.org. 